\title{
Higher-Order Numerical Method for Singularly Perturbed Delay Reaction-Diffusion Problems
}

\author{
Gemechis File Duressa, Tesfaye Aga Bullo* \\ Department of Mathematics, Jimma University, Jimma, Ethiopia \\ Email address: \\ tesfayeaga2@gmail.com (T. A. Bullo) \\ ${ }^{*}$ Corresponding author
}

To cite this article:

Gemechis File Duressa, Tesfaye Aga Bullo. Higher-Order Numerical Method for Singularly Perturbed Delay Reaction-Diffusion problems. Pure and Applied Mathematics Journal. Vol. 10, No. 3, 2021, pp. 68-76. doi: 10.11648/j.pamj.20211003.11

Received: May 18, 2021; Accepted: July 2, 2021; Published: July 9, 2021

\begin{abstract}
In this paper, a higher-order numerical method is presented for solving the singularly perturbed delay differential equations. Such kind of equations have a delay parameter on reaction term and exhibits twin boundary layers or oscillatory behavior. Recently, different numerical methods have been developed to solve the singularly perturbed delay reaction-diffusion problems. However, the obtained accuracy and its rate of convergence are satisfactory. Thus, to solve the considered problem with more satisfactory accuracy and a higher rate of convergence, the higher-order numerical method is presented. First, the given singularly perturbed delay differential equation is transformed to asymptotically equivalent singularly perturbed twopoint boundary value convection-diffusion differential equation by using Taylor series approximations. Then, the constructed singularly perturbed boundary value differential equation is replaced by three-term recurrence relation finite difference approximations. The Richardson extrapolation technique is applied to accelerate the fourth-order convergent of the developed method to the sixth-order convergent. The consistency and stability of the formulated method have been investigated very well to guarantee the convergence of the method. The rate of convergence for both the theoretical and numerical have been proven and are observed to be in accord with each other. To demonstrate the efficiency of the method, different model examples have been considered and simulation of numerical results have been presented by using MATLAB software. Numerical experimentation has been done and the results are presented for different values of the parameters. Further, The obtained numerical results described that the finding of the present method is more accurate than the findings of some methods discussed in the literature.
\end{abstract}

Keywords: Singularly Perturbed Problems, Delay Reaction-Diffusion Type, Accurate Solution, Higher-Order Method

\section{Introduction}

The particularity of noticing the relation between causes and effects arises when the cause is small and the effect is large. In the philosophy of perturbation for mathematics and physical systems, the study of this relation got a significant amount of attention in past and recent years. A singularly perturbed differential equation is a differential equation in which the highest order derivative is multiplied by a small parameter that is recognized as a perturbation parameter. The solution of singularly perturbed differential equations varies rapidly in the regions called boundary layers. The study of the solution of these equations is of great significance due to the formation of sharp boundary layers when the perturbation parameter approaches zero. Singularly perturbed delay differential equation is an equation in which the evolution of the system at a convinced time depends on the rate at an earlier time.

The delay in the process rises due to the requirement of a definite time to sense the instruction and react to it. The delay differential equation can be classified as retarded delay differential equation and neutral differential equation. The applications of delay differential equations arise in the modeling of neural variables, variational problems in control theory, description of human pupil reflex, physical and biological phenomena like optically bi-stable devices, numerical modeling in biosciences, and HIV infection [1-5].

In singular perturbation problems, when the perturbation parameter equal to zero and if the order is reduced by one then the problem is called convection-diffusion type, whereas if the 
order is reduced by two it is called reaction-diffusion type. Hence, second-order singularly perturbed delay differential equations are may be convection-diffusion or reaction-diffusion types. The former type of problem exhibits left or right boundary layer only depending on the sign of coefficient in diffusion and convection terms, while reaction-diffusion delay differential equations have dual boundary layers (both at the left and right side of the domain) or oscillatory behavior of solution depending on the sign of the sum of coefficients in reaction terms. Researchers have been developed and analyzed different numerical methods for solving singularly perturbed delay differential equations. For instance, numerical methods proposed by various authors for solving singularly perturbed delay differential equations of convection-diffusion type are; Parameter-robust numerical method based on defect-correction technique [6], Fitted mesh numerical method [7], Numerical integration method [8], using B-Spline collocation method [9], Fitted Method [10], Terminal boundary-value technique [11], Numerical integration using exponential integrating factor [12], Fitted fourth-order scheme [13] and A non-asymptotic method for general singular perturbation problems [20] so on. Further, more recently, various authors have developed numerical methods for solving singularly perturbed delay reactiondiffusion problems like; Computational method [14-17], a fourth-order numerical method [18], trigonometric B-spline [4], and Computational method for singularly perturbed delay differential equations of the reaction-diffusion type with negative shift [22].

All these works concern second-order singularly perturbed delay differential equations in which the developed methods are analyzed in different approaches and produce good accurate numerical solutions corresponding with a diverse rate of convergence to demonstrate the efficiency of the methods. However, the obtained approximate solution and the corresponding order of convergence are not more satisfactory which indicates that yet to solve singularly perturbed delay reaction-diffusion problems demands to develop of other numerical methods to produce a more accurate numerical solution. Therefore, the main objective of this paper is to present a higher-order numerical method to solve the singularly perturbed delay reaction-diffusion problems.

\section{Formulation of the Method}

Consider the singularly perturbed delay reaction-diffusion problem:

$$
\varepsilon y^{\prime \prime}(x)+a(x) y(x-\delta)+b(x) y(x)=g(x), \quad \Omega:=(0,1),
$$

subject to the interval and boundary conditions,

$$
y(x)=\phi(x),-\delta \leq x \leq 0 \text { and } y(1)=\beta,
$$

where $\varepsilon$ is perturbation parameter satisfies $0<\varepsilon<<1$ with the delay parameter $\delta=o(\varepsilon), \quad \phi(x)$ is sufficiently smooth on $[-1,0]$ and $\beta$ is a given constant which is independent of $\varepsilon$. Further, coefficient functions $a(x), b(x)$, and the source function $g(x)$ are assumed to be sufficiently differentiable functions on the closed domain $\bar{\Omega}$. The layer or oscillatory behavior of the problem under consideration is conserved for $\delta \neq 0$, but sufficiently small, depending on the sign of $a(x)+b(x)$, for all $x \in \Omega$. If $a(x)+b(x)<0$ then the solution of equations (1) and (2) exhibits two boundary layer behavior which will occur at both endpoints $x=0$ and $x=1$, while $a(x)+b(x)>0$, it exhibits oscillatory behavior, $[4,5,16]$.

Taylor's series is familiar to handle the delay term as

$$
y(x-\delta)=y(x)-\delta y^{\prime}(x)+\frac{\delta^{2}}{2} y^{\prime \prime}(x)+O\left(\delta^{3}\right) .
$$

Substituting equation (3) into equation (1), gives:

$$
\varepsilon y^{\prime \prime}(x)+p(x) y^{\prime}(x)+q(x) y(x)=f(x),
$$

under the boundary conditions

$$
y(0)=\phi(0) \text { and } y(1)=\beta,
$$

where $p(x)=\frac{-2 \varepsilon \delta a(x)}{2 \varepsilon+\delta^{2} a(x)} \quad, \quad q(x)=\frac{2 \varepsilon(a(x)+b(x))}{2 \varepsilon+\delta^{2} a(x)} \quad$ and $f(x)=\frac{2 \varepsilon g(x)}{2 \varepsilon+\delta^{2} a(x)}$.

To develop the finite difference method for the problem in equation (4), the interval $[0,1]$ is divided into $N$ subintervals with a mesh length of each $h=\frac{1}{N}$. Form this, we obtain a set of grid points, $x_{0}=0, x_{N}=1$ and $x_{i}=x_{0}+i h$, for $i=1,2, \ldots, N-1$. For convenience, let denote $p\left(x_{i}\right)=p_{i}$ $q\left(x_{i}\right)=q_{i}, \quad y\left(x_{i}\right)=y_{i}, \quad y^{\prime}\left(x_{i}\right)=y_{i}^{\prime}, \ldots, \quad y^{(n)}\left(x_{i}\right)=y_{i}^{(n)}$. Assume that $y(x)$ has continuous higher-order derivatives on $[0,1]$, using Taylor's series expansion, we have:

$$
\begin{aligned}
& y_{i+1}=y_{i}+h y_{i}^{\prime}+\frac{h^{2}}{2 !} y_{i}^{\prime \prime}+\frac{h^{3}}{3 !} y_{i}^{\prime \prime \prime}+\frac{h^{4}}{4 !} y_{i}^{(4)}+\frac{h^{5}}{5 !} y_{i}^{(5)}+\frac{h^{6}}{6 !} y_{i}^{(6)}+\ldots \\
& y_{i-1}=y_{i}-h y_{i}^{\prime}+\frac{h^{2}}{2 !} y_{i}^{\prime \prime}-\frac{h^{3}}{3 !} y_{i}^{\prime \prime \prime}+\frac{h^{4}}{4 !} y_{i}^{(4)}-\frac{h^{5}}{5 !} y_{i}^{(5)}+\frac{h^{6}}{6 !} y_{i}^{(6)}+\ldots
\end{aligned}
$$

Subtracting equation (7) from equation (6) or adding the two equations, we yield

$$
y_{i}^{\prime}=\frac{y_{i+1}-y_{i-1}}{2 h}-\frac{h^{2}}{6} y_{i}^{\prime \prime \prime}+\tau_{1}
$$

or

$$
y_{i}^{\prime \prime}=\frac{y_{i+1}-2 y_{i}+y_{i-1}}{h^{2}}-\frac{h^{2}}{12} y_{i}^{(4)}+\tau_{2}
$$

where $\tau_{1}=-\frac{h^{4}}{120} y_{i}^{(5)}$ and $\tau_{2}=\frac{-h^{4}}{360} y_{i}^{(6)}$.

Substituting equation (8) into the discrete form of equation (4) gives: 


$$
\frac{\varepsilon}{h^{2}}\left(y_{i+1}-2 y_{i}+y_{i-1}\right)+\frac{p_{i}}{2 h}\left(y_{i+1}-y_{i-1}\right)+q_{i} y_{i}-\frac{p_{i} h^{2}}{6} y_{i}^{\prime \prime \prime}-\frac{\varepsilon h^{2}}{12} y_{i}^{(4)}+\tau_{0}=f_{i}
$$

where $\tau_{0}=p_{i} \tau_{1}+\varepsilon \tau_{2}$.

Once and twice successively differentiating both sides of equation (4), concerning the independent variable and considering at the nodal point $x_{i}$, yields $y_{i}^{\prime \prime \prime}$ and $y_{i}^{(4)}$ respectively. Then substituting these values into equation (9), gives the three-term recurrence relation of the form:

$$
E_{i} y_{i-1}-F_{i} y_{i}+G_{i} y_{i+1}=H_{i}, \quad i=1,2, \ldots, N-1,
$$

where $E_{i}=\frac{\varepsilon}{h^{2}}+\frac{2 p_{i}^{\prime}+q_{i}}{12}+\frac{p_{i}^{2}}{12 \varepsilon}-\left(\frac{p_{i}}{2 h}+\frac{h}{24}\left(p_{i}^{\prime \prime}+2 q_{i}^{\prime}\right)+\frac{p_{i} h}{24 \varepsilon}\left(p_{i}^{\prime}+q_{i}\right)\right)$,

$$
\begin{gathered}
F_{i}=2\left(\frac{\varepsilon}{h^{2}}+\frac{2 p_{i}^{\prime}+q_{i}}{12}+\frac{p_{i}^{2}}{12 \varepsilon}\right)-\left(q_{i}+\frac{h^{2} q_{i}^{\prime \prime}}{12}+\frac{p_{i} q_{i}^{\prime} h^{2}}{12 \varepsilon}\right), \\
G_{i}=\frac{\varepsilon}{h^{2}}+\frac{2 p_{i}^{\prime}+q_{i}}{12}+\frac{p_{i}^{2}}{12 \varepsilon}+\left(\frac{p_{i}}{2 h}+\frac{h}{24}\left(p_{i}^{\prime \prime}+2 q_{i}^{\prime}\right)+\frac{p_{i} h}{24 \varepsilon}\left(p_{i}^{\prime}+q_{i}\right)\right), \\
H_{i}=f_{i}+\frac{p_{i} h^{2}}{12 \varepsilon} f_{i}^{\prime}+\frac{h^{2}}{12} f_{i}^{\prime \prime} .
\end{gathered}
$$

\section{Richardson Extrapolation}

The purpose of this section is to improve the accuracy and the order of convergence by convergence acceleration technique which involves a combination of two computed approximate solutions. The linear combination turns out to be a better approximation, [24, 26].

Ever since from equation (8) or equation (9), we know that the truncation error of the formulated method is $O\left(h^{4}\right)$. Hence, we have

$$
\left|y\left(x_{i}\right)-Y_{N}\right| \leq C\left(h^{4}\right)
$$

where $y\left(x_{i}\right)$ and $Y_{N}$ are exact and approximate solutions respectively, $C$ is a constant independent of mesh sizes $h$.

Let $\Omega^{2 N}$ be the mesh obtained by bisecting each mesh interval in $\Omega^{N}$ and denote the approximation of the solution on $\Omega^{2 N}$ by $Y_{2 N}$. Consider equation (10) works for any mesh size $h \neq 0$, which indicates:

$$
y\left(x_{i}\right)-Y_{N} \leq C h^{4}+R^{N}, x_{i} \in \Omega^{N} .
$$

So, it works for any mesh size $\frac{h}{2} \neq 0$ leads to

$$
y\left(x_{i}\right)-Y_{2 N} \leq C \frac{h^{4}}{16}+R^{2 N}, x_{i} \in \Omega^{2 N},
$$

where the remainders, $R^{N}$ and $R^{2 N}$ are $O\left(h^{6}\right)$.

Assume that $C$ is a constant in both equations (12) and (13), we multiply equation (13) by 16 , and then combining the two inequalities in equations (12) and (13) gives

$$
15 y\left(x_{i}\right)-\left(16 Y_{2 N}-Y_{N}\right) \leq 16 R^{2 N}-R^{N} \text {. }
$$

This can be re-written as

$$
y\left(x_{i}\right)-\frac{1}{15}\left(16 Y_{2 N}-Y_{N}\right) \leq \frac{1}{15}\left(16 R^{2 N}-R^{N}\right),
$$

and further by notation

$$
\left(Y_{N}\right)^{e x t}=\frac{1}{15}\left(16 Y_{2 N}-Y_{N}\right)
$$

is also an approximation to the exact solution $y\left(x_{i}\right)$. Hence, using the approximate solution in equation (14), and the truncation terms in equations (12) and (13), we further obtain the truncation error:

$$
\left|y\left(x_{i}\right)-\left(Y_{N}\right)^{e x t}\right| \leq C h^{6}
$$

This indicates that the Richardson extrapolation method accelerates the order of convergence from fourth-order to sixth-order.

\section{Consistency of the Method}

Local truncation error refers to the difference between the differential equation and its finite difference approximations. A finite difference scheme is consistent if the limit of truncation error (TE) is equal to zero as the mesh size $h$ goes to zero. Further, local truncation errors measure how well a finite difference discretization approximates the differential equation [19, 21, 23-26].

Truncation error (TE) from equations (8) and (9) becomes

$$
\tau_{0}=p_{i} \tau_{1}+\varepsilon \tau_{2}=-\frac{p_{i} h^{4}}{120} y_{i}^{(5)}-\frac{\varepsilon h^{4}}{360} y_{i}^{(6)}=-h^{4}\left(\frac{p_{i}}{120} y_{i}^{(5)}+\frac{\varepsilon}{360} y_{i}^{(6)}\right)
$$

then, it is possible to show that

$$
|T E|=\left|\tau_{0}\right|=\left|-h^{4}\left(\frac{p\left(\xi_{i}\right)}{120} y^{(5)}\left(\xi_{i}\right)+\frac{\varepsilon}{360} y^{(6)}\left(\xi_{i}\right)\right)\right| \leq C h^{4}, x_{i-1}<\xi_{i}<x_{i+1},
$$

where $C=\max _{x_{i-1}<\xi_{i}<x_{i+1}}\left|-\left(\frac{p\left(\xi_{i}\right)}{120} y^{(5)}\left(\xi_{i}\right)+\frac{\varepsilon}{360} y^{(6)}\left(\xi_{i}\right)\right)\right|$ is constant.

This implies that the developed method is accurate in order four. Hence, from the definition of consistency, both the truncation errors in equations (15) and (16) satisfy: 


$$
\lim _{h \rightarrow 0} T E=\lim _{h \rightarrow 0} C h^{4}=\lim _{h \rightarrow 0} C h^{6}=0 .
$$

Thus, the proposed method is consistent, (See [24]).

\section{Stability of the Method}

Consider the developed scheme in equation (10) which is given by:

$$
E_{i} y_{i-1}-F_{i} y_{i}+G_{i} y_{i+1}=H_{i} \text {. }
$$

If we multiply both sides of equation (10) by $h^{2}$ and taking a limit as $h \rightarrow 0$, the coefficient functions:

$$
\begin{aligned}
\lim _{h \rightarrow 0} E_{i} & =\lim _{h \rightarrow 0} h^{2}\left(\frac{\varepsilon}{h^{2}}+\frac{2 p_{i}^{\prime}+q_{i}}{12}+\frac{p_{i}^{2}}{12 \varepsilon}-\frac{p_{i}}{2 h}-\frac{h}{24}\left(p_{i}^{\prime \prime}+2 q_{i}^{\prime}\right)-\frac{p_{i} h}{24 \varepsilon}\left(p_{i}^{\prime}+q_{i}\right)\right) \\
& =\varepsilon+\lim _{h \rightarrow 0} h^{2}\left(\frac{2 p_{i}^{\prime}+q_{i}}{12}+\frac{p_{i}^{2}}{12 \varepsilon}-\frac{p_{i}}{2 h}-\frac{h}{24}\left(p_{i}^{\prime \prime}+2 q_{i}^{\prime}\right)-\frac{p_{i} h}{24 \varepsilon}\left(p_{i}^{\prime}+q_{i}\right)\right)=\varepsilon .
\end{aligned}
$$

and

$$
\begin{aligned}
\lim _{h \rightarrow 0} F_{i}= & 2 \lim _{h \rightarrow 0} h^{2}\left(\frac{\varepsilon}{h^{2}}+\frac{2 p_{i}^{\prime}+q_{i}}{12}+\frac{p_{i}^{2}}{12 \varepsilon}-q_{i}-\frac{h^{2} q_{i}^{\prime \prime}}{12}-\frac{p_{i} q_{i}^{\prime} h^{2}}{12 \varepsilon}\right), \\
& =2 \varepsilon+\lim _{h \rightarrow 0} h^{2}\left(\frac{2 p_{i}^{\prime}+q_{i}}{12}+\frac{p_{i}^{2}}{12 \varepsilon}-q_{i}-\frac{h^{2} q_{i}^{\prime \prime}}{12}-\frac{p_{i} q_{i}^{\prime} h^{2}}{12 \varepsilon}\right)=2 \varepsilon
\end{aligned}
$$

Likewise, $\lim _{h \rightarrow 0} G_{i}=\varepsilon$ and $\lim _{h \rightarrow 0} H_{i}=0$, for $i=1,2, \ldots, N-1$.

Hence, for sufficiently small $h$ we can get:

$$
E_{i}=G_{i}=\varepsilon, F_{i}=2 \varepsilon, \text { and } H_{i}=0, \text { for } i=1,2, \ldots, N-1 .
$$

Thus, equation (10) becomes a system of equations that can be written in a matrix form of

$$
M Y=H,
$$

where the matrices:

$$
\begin{gathered}
M=\left[\begin{array}{cccccc}
-2 \varepsilon & \varepsilon & 0 & \cdots & \ldots & 0 \\
\varepsilon & -2 \varepsilon & \varepsilon & 0 & \vdots & \vdots \\
0 & \varepsilon & -2 \varepsilon & \ddots & 0 & 0 \\
0 & 0 & \ddots & \ddots & \varepsilon & 0 \\
\vdots & \vdots & 0 & \varepsilon & -2 \varepsilon & \varepsilon \\
0 & \cdots & 0 & 0 & \varepsilon & -2 \varepsilon
\end{array}\right], Y=\left[\begin{array}{c}
-\varepsilon y_{0} \\
0 \\
\vdots \\
\vdots \\
\vdots \\
y_{2} \\
y_{N-2} \\
y_{N-1}
\end{array}\right] \\
\text { and } H=\left[\begin{array}{c}
y_{1} \\
-\varepsilon y_{N}
\end{array}\right]
\end{gathered}
$$

Hence, the matrix $M$ is irreducible.

$$
\left|E_{i}\right|>0,\left|G_{i}\right|>0 \text { and }\left|F_{i}\right| \geq\left|E_{i}\right|+\left|G_{i}\right| \text {. }
$$

This proves the diagonal dominant of $M$. Under these conditions, any tri-diagonal solver is stable for sufficiently small $h$, as shown in the book "Numerical solution of differential equations, Introduction to finite difference and finite element methods", [19]. As proved by Smith [21], the eigenvalues of a tri-diagonal $(N-1) \times(N-1)$ of matrix $M$ are:

$$
\lambda_{s}=F_{i}-2 \sqrt{E_{i} G_{i}} \cos \frac{s \pi}{N}=2 \varepsilon\left(1-\cos \frac{s \pi}{N}\right), \quad s=1,2, \ldots, N-1
$$

From trigonometric identity, $1-\cos \frac{s \pi}{N}=2 \sin ^{2} \frac{s \pi}{2 N}$, eigenvalues of matrix $M$ can be re-written as:

$$
\lambda_{s}=2 \varepsilon\left(2 \sin ^{2} \frac{s \pi}{2 N}\right)=4 \varepsilon \sin ^{2} \frac{s \pi}{2 N} \leq 4 \varepsilon
$$

A finite difference method for solving differential equations is stable if $M$ is invertible and

$$
\left\|M^{-1}\right\| \leq C, \forall 0<h<h_{0},
$$

where $C$ and $h_{0}$ are two constants that are independent of $h$, $[19,21]$.

Since matrix $M$ is symmetric also its inverse matrix $M^{-1}$ is symmetric and the eigenvalues $M^{-1}$ are given by $\frac{1}{\lambda_{s}}$, we have

$$
\left\|M^{-1}\right\|=\frac{1}{\lambda_{s}}=\frac{1}{4 \varepsilon} \leq C, \text { where } \mathrm{C} \text { is independent of } \mathrm{h} \text {. }
$$


Thus, the developed scheme in equation (10) is stable.

A consistent and stable finite difference method is convergent by Lax's equivalence theorem. Hence, as we have shown above the proposed method is satisfying the criteria for both consistency and stability which are equivalents to the convergence of the method.

Table 1. The maximum absolute errors for Example 1, when $\delta=0.5 \varepsilon$.

\begin{tabular}{|c|c|c|c|c|c|}
\hline$\varepsilon \downarrow$ & $N=2^{4}$ & $N=2^{5}$ & $N=2^{6}$ & $N=2^{7}$ & $N=2^{8}$ \\
\hline \multicolumn{6}{|c|}{ Our method } \\
\hline $2^{-4}$ & $1.2780 \mathrm{e}-08$ & $2.0643 \mathrm{e}-10$ & $3.2591 \mathrm{e}-12$ & $3.9108 \mathrm{e}-13$ & $8.5487 \mathrm{e}-15$ \\
\hline $2^{-5}$ & $9.1840 \mathrm{e}-08$ & $1.5860 \mathrm{e}-09$ & $2.5205 \mathrm{e}-11$ & $3.9610 \mathrm{e}-13$ & $1.1824 \mathrm{e}-14$ \\
\hline $2^{-6}$ & $6.8073 \mathrm{e}-07$ & $1.2266 \mathrm{e}-08$ & $1.9844 \mathrm{e}-10$ & $3.1440 \mathrm{e}-12$ & $4.9766 \mathrm{e}-14$ \\
\hline $2^{-7}$ & $4.4400 \mathrm{e}-06$ & $9.2032 \mathrm{e}-08$ & $1.5775 \mathrm{e}-09$ & $2.5078 \mathrm{e}-11$ & $3.8966 \mathrm{e}-13$ \\
\hline $2^{-8}$ & $2.0837 \mathrm{e}-05$ & $6.8129 \mathrm{e}-07$ & $1.2292 \mathrm{e}-08$ & $1.9949 \mathrm{e}-10$ & $3.1594 \mathrm{e}-12$ \\
\hline $2^{-9}$ & $4.6493 \mathrm{e}-05$ & $4.4755 \mathrm{e}-06$ & $9.2891 \mathrm{e}-08$ & $1.5859 \mathrm{e}-09$ & $2.5213 \mathrm{e}-11$ \\
\hline $2^{-10}$ & $1.3651 \mathrm{e}-04$ & $2.1066 \mathrm{e}-05$ & $6.8425 \mathrm{e}-07$ & $1.2348 \mathrm{e}-08$ & $2.0083 \mathrm{e}-10$ \\
\hline \multicolumn{6}{|c|}{ Results in [18] } \\
\hline $2^{-4}$ & $3.0267 \mathrm{e}-05$ & $1.9031 \mathrm{e}-06$ & $1.1950 \mathrm{e}-07$ & $7.4728 \mathrm{e}-09$ & $4.6725 \mathrm{e}-10$ \\
\hline $2^{-5}$ & $1.1987 \mathrm{e}-04$ & $7.8382 \mathrm{e}-06$ & $4.9134 \mathrm{e}-07$ & $3.0732 \mathrm{e}-08$ & $1.9223 \mathrm{e}-09$ \\
\hline $2^{-6}$ & $4.9863 \mathrm{e}-04$ & $3.1795 \mathrm{e}-05$ & $1.9986 \mathrm{e}-06$ & $1.2579 \mathrm{e}-07$ & $7.8649 \mathrm{e}-09$ \\
\hline $2^{-7}$ & $1.9386 \mathrm{e}-03$ & $1.2530 \mathrm{e}-04$ & $8.1293 \mathrm{e}-06$ & $5.0955 \mathrm{e}-07$ & $3.1919 \mathrm{e}-08$ \\
\hline $2^{-8}$ & $6.4424 \mathrm{e}-03$ & $5.1006 \mathrm{e}-04$ & $3.2516 \mathrm{e}-05$ & $2.0492 \mathrm{e}-06$ & $1.2889 \mathrm{e}-07$ \\
\hline $2^{-9}$ & $1.7543 \mathrm{e} 02$ & $1.9764 \mathrm{e}-03$ & $1.2772 \mathrm{e}-04$ & $8.2523 \mathrm{e}-06$ & $5.1725 \mathrm{e}-07$ \\
\hline $2^{-10}$ & $3.8002 \mathrm{e}-02$ & $6.557 \mathrm{e}-03$ & $5.1492 \mathrm{e}-04$ & $3.2824 \mathrm{e}-05$ & $2.0727 e-06$ \\
\hline
\end{tabular}

Table 2. Comparison of maximum absolute error for Example 2 at $\delta=0.5 \varepsilon$.

\begin{tabular}{|c|c|c|c|c|}
\hline$\varepsilon \downarrow$ & $h=2^{-4}$ & $h=2^{-5}$ & $h=2^{-6}$ & $h=2^{-7}$ \\
\hline \multicolumn{5}{|c|}{ Our Method } \\
\hline $2^{-4}$ & $4.4178 \mathrm{e}-10$ & $6.9629 \mathrm{e}-12$ & $1.1102 \mathrm{e}-13$ & $2.5091 \mathrm{e}-14$ \\
\hline $2^{-5}$ & $3.1135 \mathrm{e}-09$ & $4.9464 \mathrm{e}-11$ & $7.7482 \mathrm{e}-13$ & $3.5527 \mathrm{e}-14$ \\
\hline $2^{-6}$ & $2.2262 \mathrm{e}-08$ & $3.6509 \mathrm{e}-10$ & $5.7497 \mathrm{e}-12$ & $8.4266 \mathrm{e}-14$ \\
\hline $2^{-7}$ & $1.6638 \mathrm{e}-07$ & $2.8212 \mathrm{e}-09$ & $4.4965 \mathrm{e}-11$ & $7.0516 \mathrm{e}-13$ \\
\hline $2^{-8}$ & $1.2099 \mathrm{e}-06$ & $2.1836 \mathrm{e}-08$ & $3.5620 \mathrm{e}-10$ & $5.6251 \mathrm{e}-12$ \\
\hline $2^{-9}$ & $7.9665 \mathrm{e}-06$ & $1.6541 \mathrm{e}-07$ & $2.8101 \mathrm{e}-09$ & $4.4750 \mathrm{e}-11$ \\
\hline $2^{-10}$ & $3.7536 \mathrm{e}-05$ & $1.2077 \mathrm{e}-06$ & $2.1797 \mathrm{e}-08$ & $3.5540 \mathrm{e}-10$ \\
\hline \multicolumn{5}{|c|}{ Results in [18] } \\
\hline $2^{-4}$ & $4.5775 \mathrm{e}-06$ & $2.8651 \mathrm{e}-07$ & $1.7913 \mathrm{e}-08$ & $1.1197 \mathrm{e}-09$ \\
\hline $2^{-5}$ & $1.6246 \mathrm{e}-05$ & $1.0190 \mathrm{e}-06$ & $6.3830 \mathrm{e}-08$ & $3.9901 \mathrm{e}-09$ \\
\hline $2^{-6}$ & $5.9281 \mathrm{e}-05$ & $3.7757 \mathrm{e}-06$ & $2.3632 \mathrm{e}-07$ & $1.4791 \mathrm{e}-08$ \\
\hline $2^{-7}$ & $2.2949 \mathrm{e}-04$ & $1.4731 \mathrm{e}-05$ & $9.2549 \mathrm{e}-07$ & $5.7989 \mathrm{e}-08$ \\
\hline $2^{-8}$ & $9.1215 \mathrm{e}-04$ & $5.8144 \mathrm{e}-05$ & $3.6824 \mathrm{e}-06$ & $2.3104 \mathrm{e}-07$ \\
\hline $2^{-9}$ & $3.5308 \mathrm{e}-03$ & $2.2815 \mathrm{e}-04$ & $1.4669 \mathrm{e}-05$ & $9.2088 \mathrm{e}-07$ \\
\hline $2^{-10}$ & $1.1709 \mathrm{e}-02$ & $9.1043 \mathrm{e}-04$ & $5.8034 \mathrm{e}-05$ & $3.6736 \mathrm{e}-06$ \\
\hline
\end{tabular}

\section{Numerical Examples and Results}

To confirm the applicability of the formulated method, we have been realized the method on four examples, the first two with twin boundary layers, while the remaining with oscillatory behavior. Since these examples have no exact solution; consequently the numerical solutions are computed using the double mesh principle. The maximum absolute errors are calculated using the double-mesh principle before and after applying Richardson extrapolation is given by 


$$
E_{\varepsilon}^{N}=\max _{\forall x_{i} \in[0,1]}\left|Y_{2 N}-Y_{N}\right| \text {, and }
$$

$\left(E_{\varepsilon}^{N}\right)^{e x t}=\max _{\forall x_{i} \in[0,1]}\left|\left(Y_{2 N}\right)^{e x t}-\left(Y_{N}\right)^{e x t}\right|$ respectively.

The corresponding order of convergence is determined by $P_{\varepsilon}^{N}=\frac{\log E_{\varepsilon}^{N}-\log E_{\mathcal{\varepsilon}}^{2 N}}{\log 2}$.

Example 1: Consider the singularly perturbed delay differential equation

$$
\left\{\begin{array}{l}
\varepsilon y^{\prime \prime}(x)-2 y(x-\delta)-y(x)=1, \quad x \in(0,1), \\
y(x)=1,-\delta \leq x \leq 0, \\
y(1)=0 .
\end{array},\right.
$$

The maximum absolute errors are presented in Tables 1 and 3 for different values of parameters and numerical computation in graphs as Figures 1 and 2.

Example 2: Consider the differential equation

$$
\left\{\begin{array}{l}
\varepsilon y^{\prime \prime}(x)+0.25 y(x-\delta)-y(x)=1, \quad x \in(0,1), \\
y(x)=1,-\delta \leq x \leq 0, \\
y(1)=0 .
\end{array}\right.
$$

\begin{tabular}{|c|c|c|c|c|c|c|}
\hline & Example 1 & & & Example 2 & & \\
\hline$\varepsilon \downarrow$ & Our Method & Results [18] & Results [4] & Our Method & Results [18] & Results [4] \\
\hline 0.03 & $5.3152 \mathrm{e}-14$ & $8.2805 \mathrm{e}-09$ & $5.7000 \mathrm{e}-05$ & $1.9429 \mathrm{e}-14$ & $1.1784 \mathrm{e}-09$ & $3.3000 \mathrm{e}-05$ \\
\hline 0.05 & $5.8620 \mathrm{e}-14$ & $7.7093 e-09$ & $3.9000 \mathrm{e}-05$ & $2.4869 \mathrm{e}-14$ & $1.1796 \mathrm{e}-09$ & $2.5000 \mathrm{e}-05$ \\
\hline 0.09 & $7.9575 \mathrm{e}-14$ & $6.0459 \mathrm{e}-09$ & $7.1000 \mathrm{e}-03$ & $1.9429 \mathrm{e}-14$ & $1.1790 \mathrm{e}-09$ & $6.0000 \mathrm{e}-06$ \\
\hline
\end{tabular}

Table 3. Comparison of maximum absolute error obtained for Example 1 and 2 at different values of $\delta$ with $\varepsilon=0.1$ and the number of intervals $N=100$.
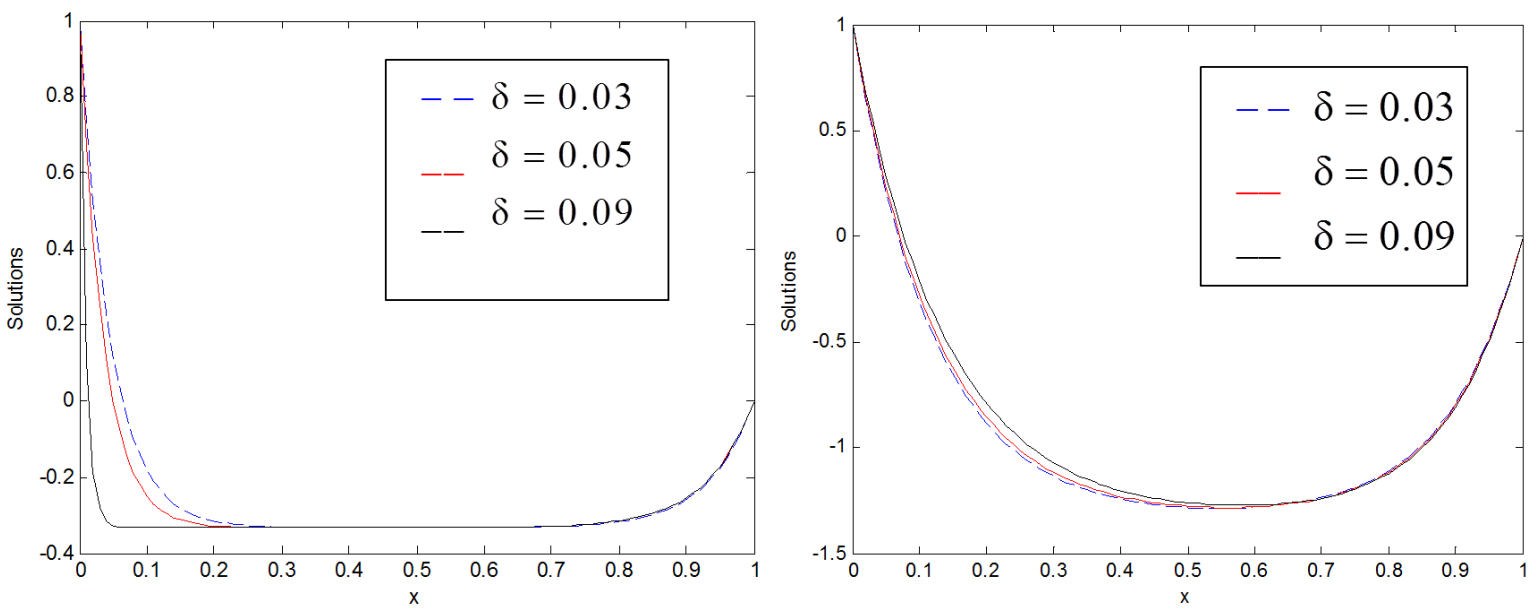

Figure 1. The behavior of the numerical solution of Example 1 on the left side and for Example 2 in the right adjacent with $\varepsilon=0.01$ and $N=100$.
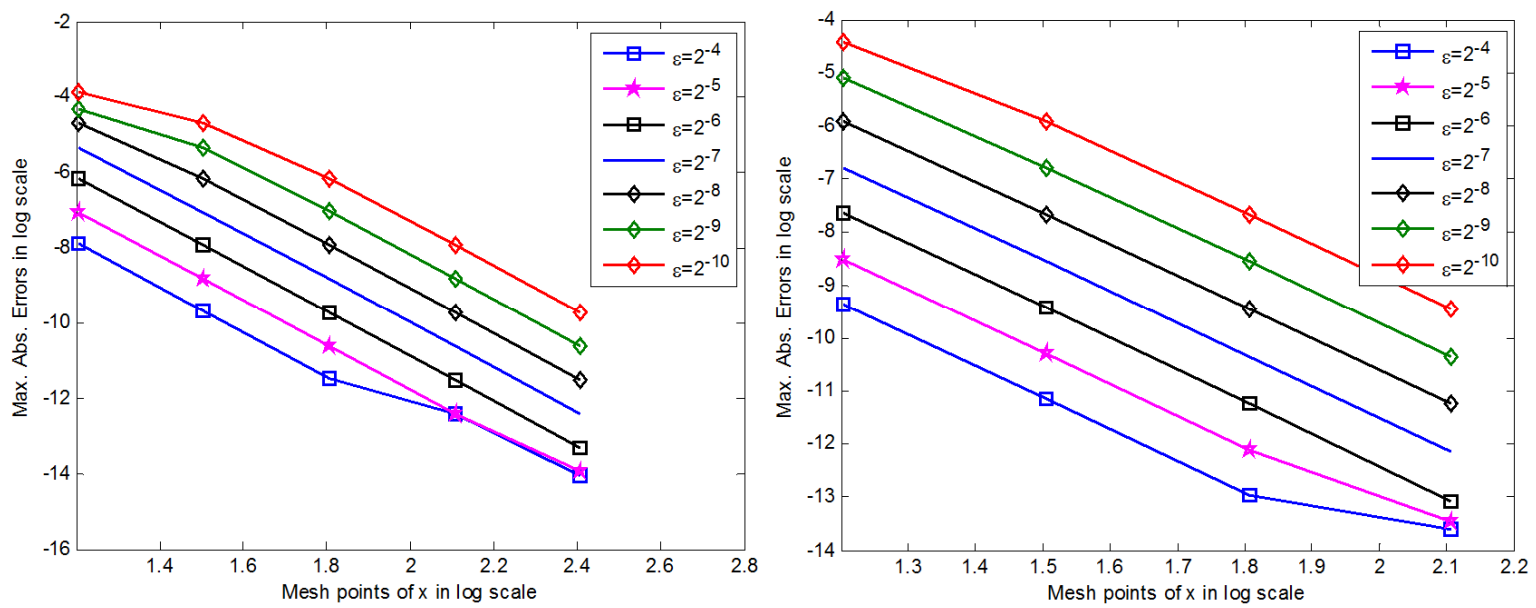

Figure 2. Effects of perturbation parameter on the numerical solution by log-log plot for Example 1 on the left side and Example 2 in the right adjacent when $\delta=0.5 \varepsilon$ and $h=\left\{2^{-4}, 2^{-5}, 2^{-6}, 2^{-7}, 2^{-8}\right\}$.

Comparisons of maximum absolute errors are presented in Tables 2 and 3 with numerical computation in graphs as Figures 1 and 2 . 
Example 3: Consider the differential equation

$$
\left\{\begin{array}{l}
\varepsilon y^{\prime \prime}(x)+0.25 y(x-\delta)+y(x)=1, \quad x \in(0,1) \\
y(x)=1,-\delta \leq x \leq 0 \\
y(1)=0
\end{array}\right.
$$

The maximum absolute errors are presented in Tables 4 and 7 with the corresponding rate of convergence given in Table 6 and graphical representation in Figures 3 and 4.

Example 4: Consider the delay differential equation

$$
\left\{\begin{array}{l}
\varepsilon y^{\prime \prime}(x)+y(x-\delta)+2 y(x)=1, \quad x \in(0,1), \\
y(x)=1,-\delta \leq x \leq 0, \\
y(1)=0
\end{array}\right.
$$

The maximum absolute errors are presented in Tables 5 and 7 with the corresponding rate of convergence given in Table 6 and graphical representation in Figures 3 and 4.

Table 4. Maximum absolute error obtained for Example 3 when $\varepsilon=0.01$.

\begin{tabular}{llllll}
\hline $\boldsymbol{\delta} \downarrow \boldsymbol{N} \rightarrow$ & $\mathbf{1 6}$ & $\mathbf{3 2}$ & $\mathbf{6 4}$ & $\mathbf{1 2 8}$ & $\mathbf{2 5 6}$ \\
\hline After extrapolation & & & & & \\
0.003 & $5.1520 \mathrm{e}-06$ & $6.7087 \mathrm{e}-08$ & $9.9836 \mathrm{e}-10$ & $1.5368 \mathrm{e}-11$ & $2.0384 \mathrm{e}-13$ \\
0.005 & $5.1375 \mathrm{e}-06$ & $6.6873 \mathrm{e}-08$ & $9.9494 \mathrm{e}-10$ & $1.5471 \mathrm{e}-11$ & $2.5174 \mathrm{e}-13$ \\
0.009 & $5.0936 \mathrm{e}-06$ & $6.6274 \mathrm{e}-08$ & $9.8600 \mathrm{e}-10$ & $1.5221 \mathrm{e}-11$ & $2.8461 \mathrm{e}-13$ \\
Before extrapolation & & & & & \\
0.003 & $4.0531 \mathrm{e}-03$ & $2.4849 \mathrm{e}-04$ & $1.5468 \mathrm{e}-05$ & $9.6579 \mathrm{e}-07$ & $6.0354 \mathrm{e}-08$ \\
0.005 & $4.0411 \mathrm{e}-03$ & $2.4775 \mathrm{e}-04$ & $1.5422 \mathrm{e}-05$ & $9.6292 \mathrm{e}-07$ & $6.0168 \mathrm{e}-08$ \\
0.009 & $4.0095 \mathrm{e}-03$ & $2.4582 \mathrm{e}-04$ & $1.5302 \mathrm{e}-05$ & $9.5543 \mathrm{e}-07$ & $5.9700 \mathrm{e}-08$ \\
\hline
\end{tabular}

Table 5. Maximum absolute error obtained for Example 4 when $\varepsilon=0.01$.

\begin{tabular}{llllll}
\hline $\boldsymbol{\delta} \downarrow \boldsymbol{N} \rightarrow$ & $\mathbf{1 6}$ & $\mathbf{3 2}$ & $\mathbf{6 4}$ & $\mathbf{1 2 8}$ & $\mathbf{2 5 6}$ \\
\hline After extrapolation & & & & & \\
0.003 & $1.2065 \mathrm{e}-04$ & $1.2945 \mathrm{e}-06$ & $1.8012 \mathrm{e}-08$ & $2.7472 \mathrm{e}-10$ & $4.2626 \mathrm{e}-12$ \\
0.005 & $1.1798 \mathrm{e}-04$ & $1.2843 \mathrm{e}-06$ & $1.7935 \mathrm{e}-08$ & $2.7429 \mathrm{e}-10$ & $4.2066 \mathrm{e}-12$ \\
0.009 & $1.2436 \mathrm{e}-04$ & $1.2530 \mathrm{e}-06$ & $1.7722 \mathrm{e}-08$ & $2.7178 \mathrm{e}-10$ & $4.2570 \mathrm{e}-12$ \\
Before extrapolation & & & & & \\
0.003 & $2.8956 \mathrm{e}-02$ & $1.8301 \mathrm{e}-03$ & $1.1350 \mathrm{e}-04$ & $7.1362 \mathrm{e}-06$ & $4.4576 \mathrm{e}-07$ \\
0.005 & $2.8841 \mathrm{e}-02$ & $1.8350 \mathrm{e}-03$ & $1.1350 \mathrm{e}-04$ & $7.1458 \mathrm{e}-06$ & $4.4635 \mathrm{e}-07$ \\
0.009 & $2.8479 \mathrm{e}-02$ & $1.8380 \mathrm{e}-03$ & $1.1370 \mathrm{e}-04$ & $7.1381 \mathrm{e}-06$ & $4.4588 \mathrm{e}-07$ \\
\hline
\end{tabular}

\begin{tabular}{|c|c|c|c|c|c|}
\hline & $\delta \downarrow N \rightarrow$ & 16 & 32 & 64 & 128 \\
\hline & After extra & & & & \\
\hline \multirow[t]{8}{*}{ Example 3} & 0.003 & 6.2630 & 6.0703 & 6.0216 & 6.2363 \\
\hline & 0.005 & 6.2635 & 6.0707 & 6.0070 & 5.9415 \\
\hline & 0.009 & 6.2641 & 6.0707 & 6.0175 & 5.7409 \\
\hline & Before ext & & & & \\
\hline & 0.003 & 4.0278 & 4.0058 & 4.0014 & 4.0002 \\
\hline & 0.005 & 4.0278 & 4.0058 & 4.0014 & 4.0003 \\
\hline & 0.009 & 4.0277 & 4.0058 & 4.0014 & 4.0003 \\
\hline & After extra & & & & \\
\hline \multirow[t]{7}{*}{ Example 4} & 0.003 & 6.5423 & 6.1673 & 6.0349 & 6.0101 \\
\hline & 0.005 & 6.5214 & 6.1621 & 6.0309 & 6.0269 \\
\hline & 0.009 & 6.6330 & 6.1437 & 6.0270 & 5.9965 \\
\hline & Before ext & & & & \\
\hline & 0.003 & 3.9839 & 4.0112 & 3.9914 & 4.0008 \\
\hline & 0.005 & 3.9743 & 4.0150 & 3.9895 & 4.0008 \\
\hline & 0.009 & 3.9537 & 4.0148 & 3.9935 & 4.0008 \\
\hline
\end{tabular}

Table 6. Rate of convergence for Example 3 and Example 4, when $\varepsilon=0.01$. 
Table 7. Comparison of maximum absolute error obtained for Example 3 and 4, when $\mathcal{E}=0.1$ and the number of intervals $N=100$.

\begin{tabular}{cllllll}
\hline & Example 3 & & Example 4 & & \\
\hline $\boldsymbol{\delta} \downarrow$ & Our Method & Results [18] & Results [4] & Our Method & Results [18] & Results [4] \\
\hline 0.03 & $7.7183 \mathrm{e}-13$ & $4.0323 \mathrm{e}-08$ & $1.1800 \mathrm{e}-03$ & $1.5027 \mathrm{e}-10$ & $1.5160 \mathrm{e}-07$ & $8.8000 \mathrm{e}-004$ \\
0.05 & $2.9976 \mathrm{e}-13$ & $3.9610 \mathrm{e}-08$ & $1.0400 \mathrm{e}-03$ & $1.5027 \mathrm{e}-10$ & $1.5697 \mathrm{e}-07$ & $7.6000 \mathrm{e}-004$ \\
0.09 & $1.3767 \mathrm{e}-13$ & $3.8377 \mathrm{e}-08$ & $1.0300 \mathrm{e}-03$ & $1.5027 \mathrm{e}-10$ & $1.7120 \mathrm{e}-07$ & $8.3000 \mathrm{e}-004$ \\
\hline
\end{tabular}
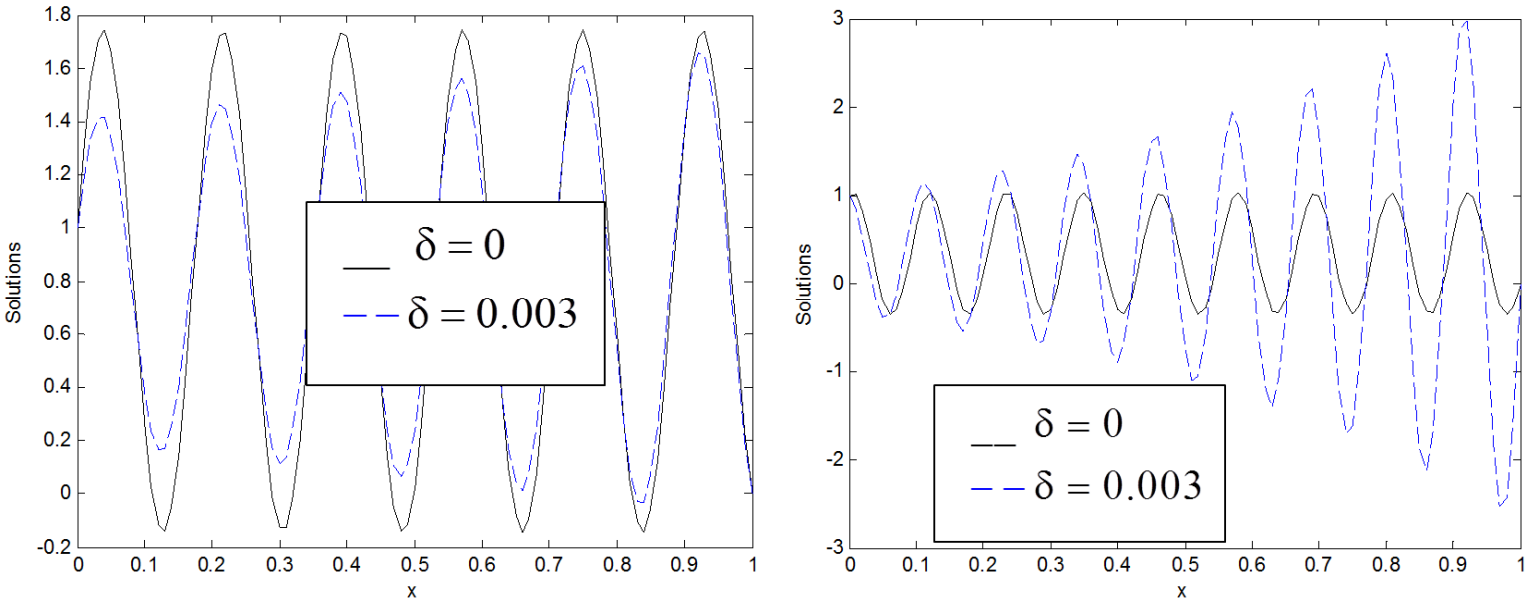

Figure 3. The behavior of the numerical solution of Example 3 on the left side and for Example 4 in the right adjacent when $\varepsilon=0.001$ and $N=100$.
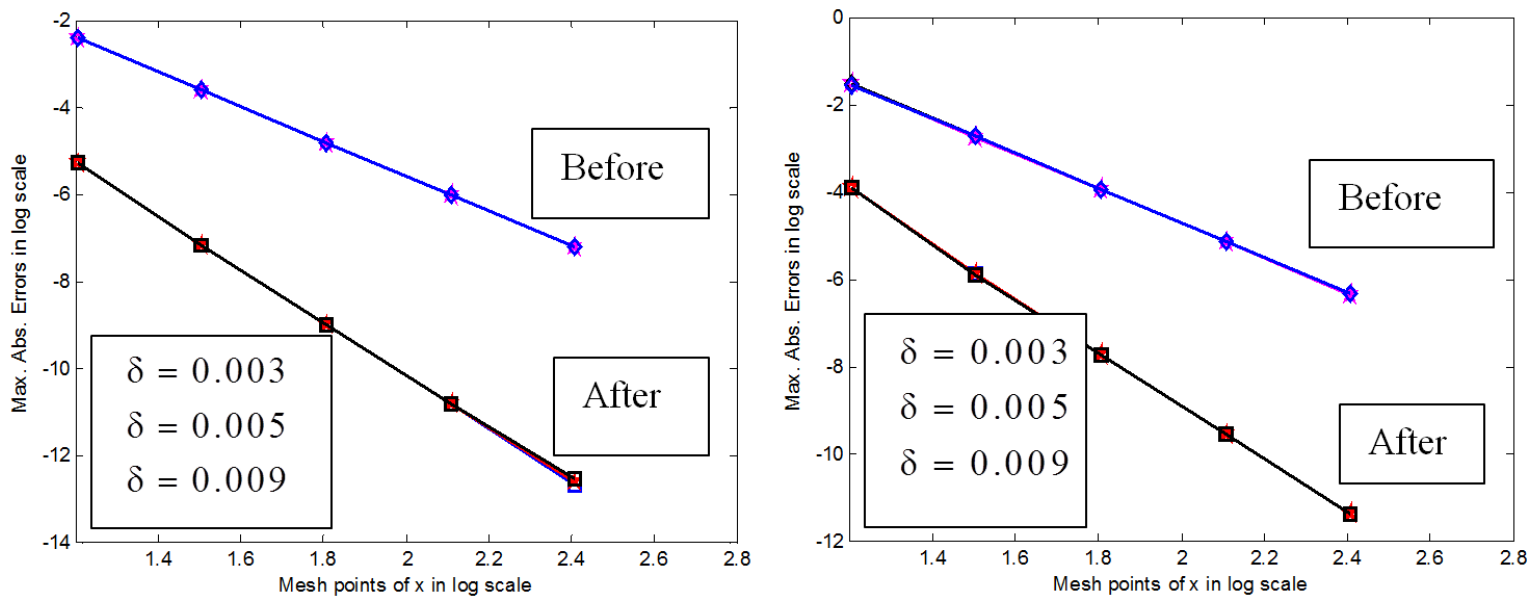

Figure 4. Effects of using before and after the Richardson extrapolation on the numerical solution of Example 3 on the left side and for Example 4 on the right adjacent with $\mathcal{E}=0.01$ and $N=\{16,32,64,128,256\}$.

\section{Discussion and Conclusion}

Numerical solution of the second-order singularly perturbed delay reaction-diffusion equations that exhibits boundary layer or oscillatory behavior via higher-order numerical method have been presented. To achieve the higher-order method, the fourth-order finite difference method is accelerated to the sixth-order one using the Richardson extrapolation technique. The efficiency of the method is validated by numerical examples and results for different parameters. The obtained numerical results have been compared with the results obtained by the methods in "Solution of second-order singular perturbed delay differential equation using Trigonometric B-Spline" and "Exponentially Fitted Numerical Method for Singularly Perturbed Differential-Difference Equations", [4, 18], (See
Tables 1, 2, 3, 7). Numerical confirmation to the contribution of applying the Richardson extrapolation technique is presented in Tables 4, 5, 6. Besides, the obtained maximum absolute errors decrease rapidly as the number of mesh points $N$ increases which indicates the convergence of the formulated method. Also, the consistency and stability of the method were investigated to guarantee convergence analysis.

Furthermore, Figures 1, 2, 3 demonstrate the effects of the parameters on the numerical solutions. Figure 4, to verify that the consequence before and after applying Richardson extrapolation and effect of decreasing number of mesh sizes of the domain on the numerical solution in case of different delay parameters and the number of mesh points. Accordingly, after applying Richardson extrapolation accuracy of the solution improved with an accelerated rate of convergence, and as the number of mesh point's $N$ increases, the accuracy of the numerical solution increases. 
Overall, a higher-order numerical method for solving the singularly perturbed delay differential equation is presented. This method is stable, consistent, and produces a more accurate solution than some existing methods for the differential equation under consideration $[4,18]$. The interested researcher will be formulating the eighth or higher-order convergent numerical methods to obtain a more accurate solution.

\section{References}

[1] FA. Rihan, "Delay differential equations in biosciences: Parameter estimation and sensitivity analysis", Proceedings of the 2013 International Conference on Applied Mathematics and Computational Methods, 2013.

[2] R. B. Stein, "Some models of neuronal variability, From the University Laboratory of Physiology", Oxford, England. Biophysical journal., 7 (1): 37, 1967.

[3] G. A., Bocharov, and F. A. Rihan, "Numerical modeling in biosciences using delay differential equations", Journal of Computational and Applied Mathematics, 125 (1): 183-199, 2000.

[4] MK. Vaid, G. Arora, "Solution of second-order singular perturbed delay differential equation using Trigonometric BSpline", International Journal of Mathematical, Engineering and Management Sciences, Vol. 4 (2), 349-360, 2019.

[5] G. Gadisa, G. File and T. Aga, "Fourth-order numerical method for singularly perturbed delay differential equations", International Journal of Applied Science and Engineering, 15 (1): 17-32, 2018.

[6] J. Mohapatra and S. Natesan, "The parameter-robust numerical method based on a defect-correction technique for singularly perturbed delay differential equations with layer behavior", International Journal of Computational Methods, 7 (4), 573-594, 2010.

[7] P. Rai and KK. Sharma, "Fitted mesh numerical method for singularly perturbed delay differential turning point problems exhibiting boundary layers", International Journal of Computer Mathematics, Vol. 89 (7), 944-961, 2012.

[8] Y. N. Reddy, G. Soujanya, and K. Phaneendra, "Numerical integration method for singularly perturbed delay differential equations", International Journal of Applied Science and Engineering, Vol. 10, 3: 249-261, 2012.

[9] D. Kumar and M. K. Kadalbajoo, "Numerical treatment of singularly perturbed delay differential equations using BSpline collocation method on Shishkin mesh", Journal of Numerical Analysis, Industrial and Applied Mathematics, vol. 7, no. 3-4, 73-90, 2012.

[10] A. Andargie and Y. N. Reddy, "Solving singularly perturbed differential-difference equations via fitted method", Applications and Applied Mathematics: An International Journal (AAM), Vol. 8, (1). 318 -332, 2013.

[11] G. File and Y. N. Reddy, "Terminal boundary-value technique for solving singularly perturbed delay differential equations", Journal of Taibah University for Science, Vol. 8, 289-300, 2014.

[12] L. S. Challa and Y. N. Reddy, "Numerical integration of singularly perturbed delay differential equations using exponential integrating factor", Math. Commun., Vol. 22, 251-264, 2017.
[13] G. Gadisa and G. File, "Fitted Fourth Order Scheme for Singularly Perturbed Delay Order Scheme for Singularly Perturbed", Ethiop. J. Educ. \& Sci. Vol. 14 (2), 102-118, 2019.

[14] D. K. Swamy, K. Phaneendra, A. B. Babu, Y. N. Reddy, "Computational method for singularly perturbed delay differential equations with twin layers or oscillatory behavior", Ain Shams Engineering Journal, Vol. 6, 391-398, 2015.

[15] G. B. Soujanya and Y. N. Reddy, "Computational method for singularly perturbed delay differential equations with a layer or oscillatory behavior", Applied Mathematics and Information Sciences, 10, (2), 527-536, 2016.

[16] G. File, G. Gadisa, T. Aga, Y. N. Reddy, "Numerical solution of singularly perturbed delay reaction-diffusion equations with a layer or oscillatory behavior", American Journal of Numerical Analysis, Vol. 5 (1), 1-10, 2017.

[17] C. L. Sirisha and Y. N. Reddy, "Solution of singularly perturbed delay differential equations with dual-layer behavior using numerical integration", Transactions on Mathematics, eISSN: 2224-2880. 2017.

[18] HG. Debela, SB. Kejela, and AD. Negassa, Exponentially Fitted Numerical Method for Singularly Perturbed Differential-Difference Equations, Hindawi, International Journal of Differential Equations, Vol. 2020, Article ID 5768323, https://doi.org/10.1155/2020/5768323.

[19] L. Zhilin, Q. Zhonghua, T. Tang, "Numerical solution of differential equations, Introduction to finite difference and finite element methods", printed in the United Kingdom by Clays, 2018.

[20] MK. Kadalbajoo, YN. Reddy, "A non-asymptotic method for general singular perturbation problems", Journal of Optimization and Applications, 55, 256- 269, 1986.

[21] G. D. Smith, "Numerical solution of partial differential equations, Finite difference methods", Third Edition, Oxford University Press, 1985.

[22] G. G. Kiltu,, G. F. Duressa, \& T. A. Bullo, Computational method for singularly perturbed delay differential equations of the reaction-diffusion type with the negative shift. Journal of Ocean Engineering and Science, 2021.

[23] T. A. Bullo, G. F. Duressa, \& G. A. Degla, Robust finite difference method for singularly perturbed two-parameter parabolic convection-diffusion problems. International Journal of Computational Methods, 18 (2), 2050034, 2021.

[24] M. K. Siraj, G. F. Duressa, \& T. A. Bullo, Fourth-order stable central difference with Richardson extrapolation method for second-order self-adjoint singularly perturbed boundary value problems. Journal of the Egyptian Mathematical Society, 27 (1), 1-14, 2019.

[25] G. R. Kusi, T. A. Bullo, \& G. F. Duressa, Quartic Nonpolynomial Spline Method for Singularly Perturbed Differential-difference Equation with Two Parameters. Journal of Engineering Advancements, 71-77, 2021.

[26] T. A. Bullo, G. F. Duressa, \& G. Degla, Accelerated fitted operator finite difference method for singularly perturbed parabolic reaction-diffusion problems. Computational Methods for Differential Equations, 9 (3), 886-898, 2021. 